\title{
The role of physical activity in the management of impaired glucose tolerance: a systematic review
}

\author{
T. Yates $\cdot$ K. Khunti $\cdot$ F. Bull $\cdot$ T. Gorely $\cdot$ M. J. Davies
}

Received: 15 December 2006 / Accepted: 23 January 2007 / Published online: 6 April 2007

(C) Springer-Verlag 2007

\begin{abstract}
Although physical activity is widely reported to reduce the risk of type 2 diabetes in individuals with prediabetes, few studies have examined this issue independently of other lifestyle modifications. The aim of this review is to conduct a systematic review of controlled trials to determine the independent effect of exercise on glucose levels and risk of type 2 diabetes in people with prediabetes (IGT and/or IFG). A detailed search of MEDLINE (19662006) and EMBASE (1980-2006) found 279 potentially relevant studies, eight of which met the inclusion criteria for this review. All eight studies were controlled trials in individuals with impaired glucose tolerance. Seven studies used a multi-component lifestyle intervention that included exercise, diet and weight loss goals and one used a structured exercise training intervention. Four studies used the incidence of diabetes over the course of the study as an outcome variable and four relied on 2-h plasma glucose as an outcome measure. In the four studies that measured the incidence of diabetes as an outcome, the risk of diabetes was reduced by approximately $50 \%$ (range $42-63 \%$ ); as
\end{abstract}

these studies reported only small changes in physical activity levels, the reduced risk of diabetes is likely to be attributable to factors other than physical activity. In the remaining four studies, only one reported significant improvements in 2-h plasma glucose even though all but one reported small to moderate increases in maximal oxygen uptake. These results indicate that the contribution of physical activity independent of dietary or weight loss changes to the prevention of type 2 diabetes in people with prediabetes is equivocal.

Keywords Exercise · IFG · Impaired fasting glucose · IGT · Impaired glucose tolerance - Physical activity - Prediabetes . Prevention · Type 2 diabetes
Abbreviations
ADA American Diabetes Association
DPP Diabetes Prevention Program
FDPS Finnish Diabetes Prevention Study
$\mathrm{MeSH}$ medical search headings

T. Yates $(\bowtie) \cdot F$. Bull $\cdot$ T. Gorely

School of Sports and Exercise Sciences,

Loughborough University,

Ashby Road,

Loughborough, Leicestershire LE11 3TU, UK

e-mail: t.e.yates@lboro.ac.uk

\section{K. Khunti}

Department of Health Sciences, University of Leicester,

Leicester, UK

\section{J. Davies}

Department of Cardiovascular Sciences, University of Leicester, Leicester, UK

\section{Introduction}

Given the growing prevalence of diabetes and the high economic cost of treating the condition and its comorbities, it is important to find effective ways of targeting those who are most at risk of developing the disease [1]. Prediabetes is the collective term for people with IGT and/or IFG [2]. Prediabetes is associated with an increased risk of development of type 2 diabetes [3] and cardiovascular disease [4-6]. There is good evidence from cross-sectional and longitudinal studies for a link between levels of physical activity and the risk of type 2 diabetes [7-9]. However, 
evidence from intervention studies in high-risk populations is limited, making it difficult to quantify the effectiveness of physical activity in reducing the risk of type 2 diabetes in individuals with prediabetes. Lifestyle intervention studies that have encouraged weight loss through a combination of dietary change and increased physical activity have reduced the risk of type 2 diabetes in individuals with IGT [10-14]. However, because physical activity was not usually analysed independently of other variables, such as weight loss, it is difficult to determine the effectiveness of physical activity at protecting against the risk of diabetes in individuals with prediabetes. Therefore, the aim of this systematic review is to establish the effectiveness of physical activity independent of other variables at reducing the risk of diabetes or improving glucose parameters in people with prediabetes.

\section{Materials and methods}

Search strategy MEDLINE (1966 to February week 4, 2006) and EMBASE (1980 to week 8, 2006) were searched for articles examining the effect of an exercise or lifestyle intervention on individuals with prediabetes. The search was carried out using medical search headings (MeSH) and by searching titles and abstracts for relevant words. For example, studies including individuals with prediabetes were found by using the MeSH 'prediabetic state,' 'insulin resistance,' 'glucose intolerance' and 'diabetes mellitus' (subheading 'prevention and control'), and by searching titles and abstracts for 'prediabetes,' 'impaired glucose tolerance,' 'IGT,' 'impaired fasting glucose' and 'IFG.' Studies that included an exercise intervention were found by using the MeSH 'lifestyle,' 'sports,' 'exercise therapy' and 'physical fitness,' and by searching titles and abstracts for 'exercise,' 'physical activity,' 'physical fitness,' 'resistance training,' 'strength training,' 'circuit training,' 'endurance training' and 'aerobic training.' In addition, the reference lists of relevant published original articles and reviews were hand-searched.

One reviewer (T. Yates) performed the electronic and hand-searches and reviewed the results. Studies that clearly did not meet the inclusion criteria were rejected during the initial review. Where uncertainty existed, the full text of the article was obtained and reviewed. Two reviewers (T. Yates and K. Khunti) independently assessed all potentially relevant studies and performed data extraction. Disagreement was resolved by discussion and, where necessary, third party adjudication.

Subjects Participants were adults (age $\geq 18$ years) diagnosed with prediabetes. Prediabetes was defined as IGT and/or IFG using one of the sets of criteria previously recommended by the WHO $[15,16]$ or the American Diabetes Association (ADA) $[17,18]$. Studies that defined IGT or IFG using other criteria were included if the mean value of the participants' plasma glucose fell within the range of IGT or IFG as defined by the WHO or ADA criteria (2-h plasma glucose $\geq 7.8 \mathrm{mmol} / 1$ and $<11.1 \mathrm{mmol} / 1$, and fasting glucose $<7.8$ $\mathrm{mmol} / \mathrm{l}$ for IGT; 2 -h plasma glucose $<7.8 \mathrm{mmol} / \mathrm{l}$, and fasting glucose $\geq 5.6 \mathrm{mmol} / 1$ and $<7.0 \mathrm{mmol} / \mathrm{l}$ for IFG).

Interventions Interventions that included an exercise programme were included. 'Exercise programme' was taken to mean any intervention that actively promoted and supported physical activity or a structured exercise training regimen. Studies that only provided individuals with brief written or verbal physical activity advice were excluded. Studies investigating the effect of a single or acute episode of exercise were also excluded.

Outcome measures Only studies with an outcome measure of physical activity and a relevant clinical measure were included. A relevant clinical measure was defined as progression to diabetes or a suitable measure of plasma glucose (2-h plasma glucose for IGT, or fasting glucose for IFG).

Type of study Randomised and non-randomised controlled trials were included.

Analysis As the heterogeneity of the type of exercise interventions and outcome measures did not lend itself to quantitative methods of analysis, a systematic narrative review was undertaken. Baseline and follow-up exercise, body mass and glucose parameters were reported using mean \pm SEM, or median (interquartile range). Results reporting the SD or the 95\% CI were converted to SEM using the formulas SEM = $\mathrm{SD} / \sqrt{ } \mathrm{n}$ and $\mathrm{SEM}=\left(\mathrm{CI}_{\text {upper }}-\mathrm{CI}_{\text {lower }}\right) / 2 t$ (where $t$ is the $t$ distribution value for a $95 \% \mathrm{CI}$ ), respectively. Where the SEM, SD or $95 \%$ CI for the change from baseline to follow-up was not reported, only the mean value is reported because of the potential error involved in calculating SEM for this figure. When available, the relative risk of diabetes in the intervention group compared with the control group was also reported.

\section{Results}

The search produced 307 hits, from which 279 potential studies were identified, of these, eight trials met the criteria for inclusion (see Fig. 1). Study details for the eight included studies are shown in Table 1; the main outcomes are presented in Table 2 . 


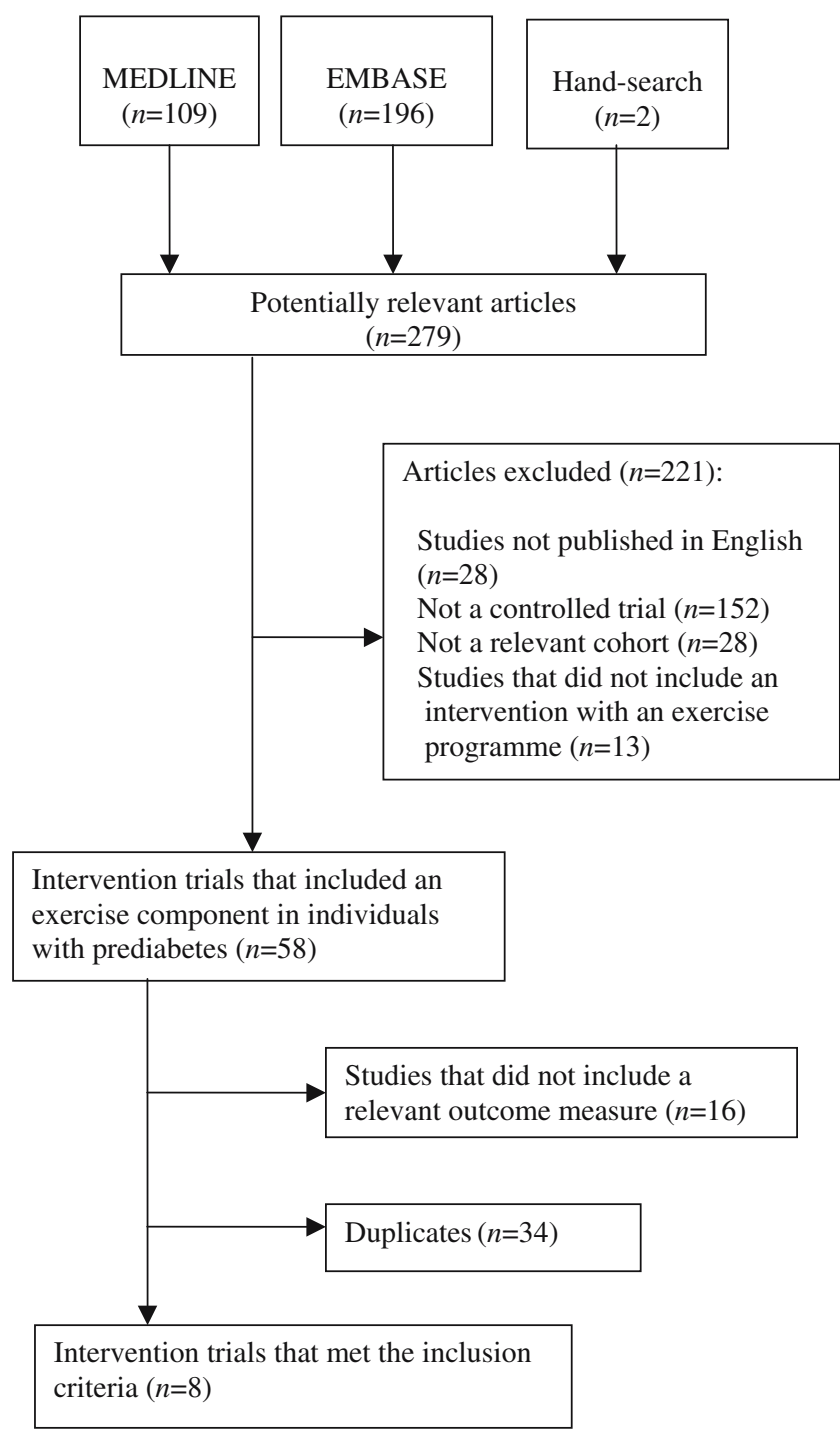

Fig. 1 Flow diagram of the literature search. Duplicates: where several studies reported on the same trial and cohort, only one published study for each trial was included for the purposes of this review; the included studies were those that reported on the cohort as a whole, had relevant follow-up measures and included the most recently published data. Where a relevant study was identified in more than one publication, the study was included only once

Study design Seven of the eight trials involved randomisation of subjects to a treatment group or control group [10,12, 1923]. The non-randomised trial identified control participants by using individuals who, for various (unstated) reasons, were not enrolled in the intervention programme [24].

Sample size Sample size ranged from 62 to 2,161. Two studies reported a power calculation based on the expected difference in the incidence of diabetes between groups [12, 21], and one reported a power calculation based on the expected difference between groups in the proportion of individuals with IGT at the end of the study [19]. In the latter study, Oldroyd et al. calculated that a total of 100 participants were required to detect a $0.6 \mathrm{mmol} / 1$ difference in fasting glucose and a $20 \%$ difference in the number of individuals with IGT, allowing for a $90 \%$ power at a significance of 0.05 . Three studies had sample sizes of fewer than 100 participants at follow-up [19, 20, 22].

Inclusion criteria All studies examined in this review included individuals with IGT and excluded those with isolated IFG [10, 12, 19-24].

Sex Except for one trial that involved only men $(n=188)$ [24], all trials included both men and women. In the included studies a total of $40 \%$ of participants were men.

Intervention conditions Seven of the eight included studies used a multi-component lifestyle intervention [10, 12, 19, 21-24], and one used a structured gym-based exercise training intervention [20].

Six of the lifestyle intervention studies were based on encouraging individuals to increase their physical activity to approximately $150 \mathrm{~min}$ of exercise of moderate to vigorous intensity per week whilst also encouraging weight loss through a healthy energy-restricted diet $[10,12,19,21$, 22, 24]. Participants in all six studies received regular encouragement and counselling from a trained dietician at least once every 3 months throughout the duration of the intervention. Two of the six studies also provided participants with the option of attending supervised exercise classes for some or all of the study duration $[21,24]$ and one provided discounted access to local gyms [19]. One study determined the effect of diet and exercise separately and in combination [10].

One lifestyle intervention included an initial 1-month stay at a wellness centre where individuals were provided with healthy dietary options and encouraged to take part in $2.5 \mathrm{~h} /$ day of light to moderate intensity exercise using the leisure facilities provided [23]. After the stay at the wellness centre, participants were encouraged to make plans about how they could incorporate healthier habits into everyday life and then received no further contact until follow-up.

The structured exercise intervention study used a training protocol of $180 \mathrm{~min}$ per week of aerobic exercise at $70 \%$ of heart rate reserve [20]. Exercise training was supervised for the first 6 months and both groups were encouraged to eat a healthy energy-balanced diet, with those in the exercise training group also being encouraged to eat a diet with a high percentage of energy from carbohydrate [20].

Outcomes Four studies included the incidence of diabetes as the main outcome [10, 12, 21, 24], and four used 2-h plasma glucose levels as a direct measure of glucose control [19, 


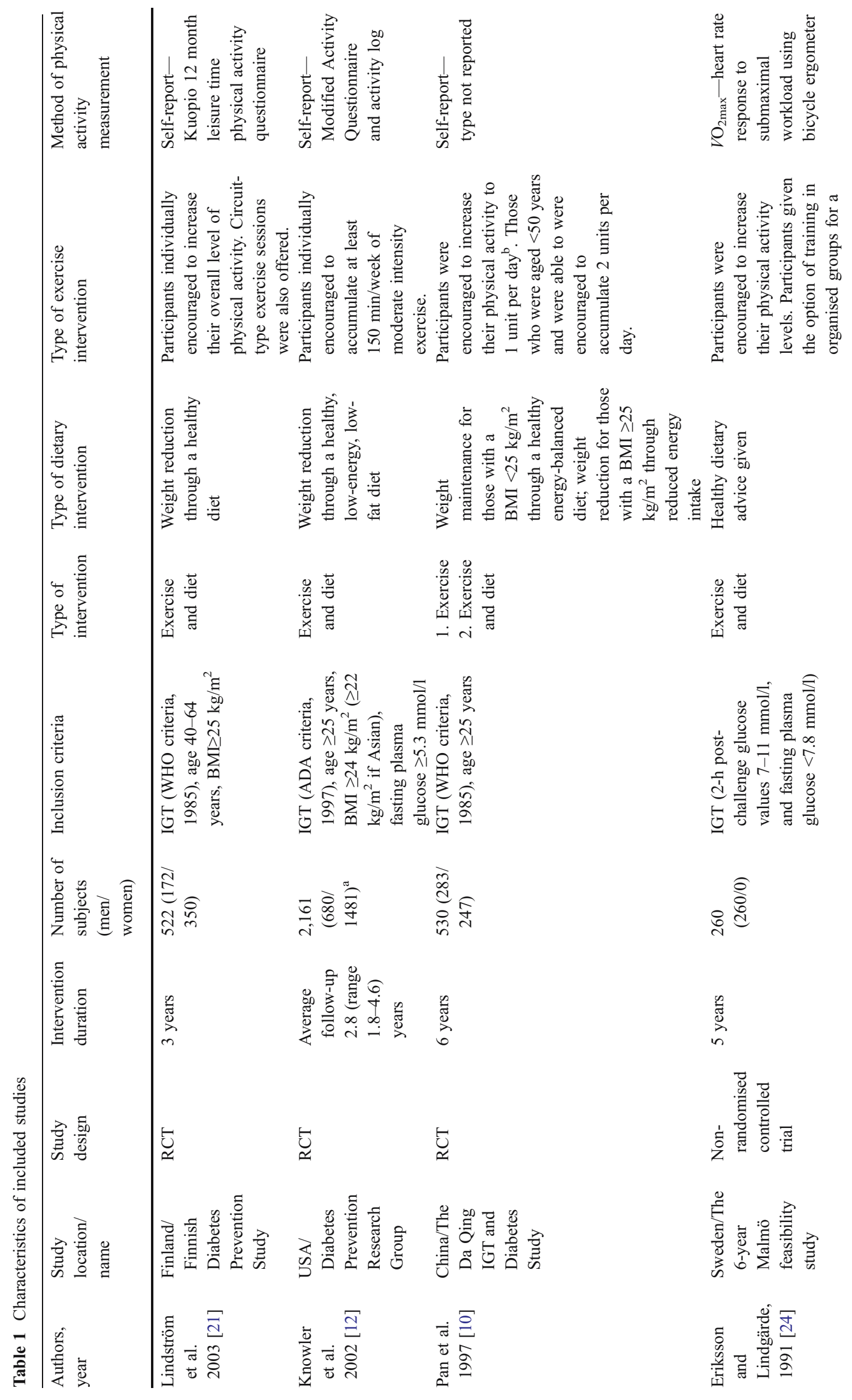




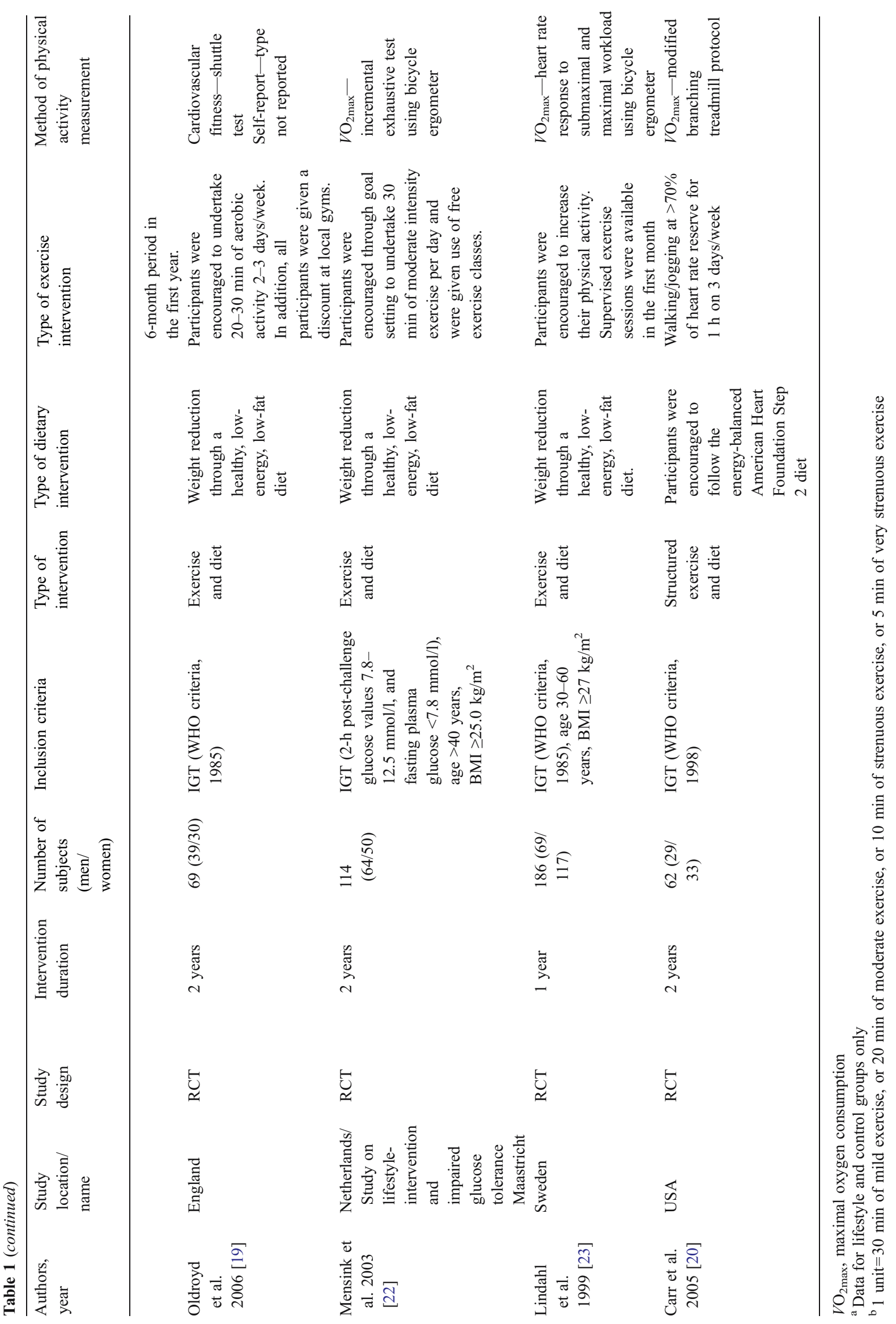




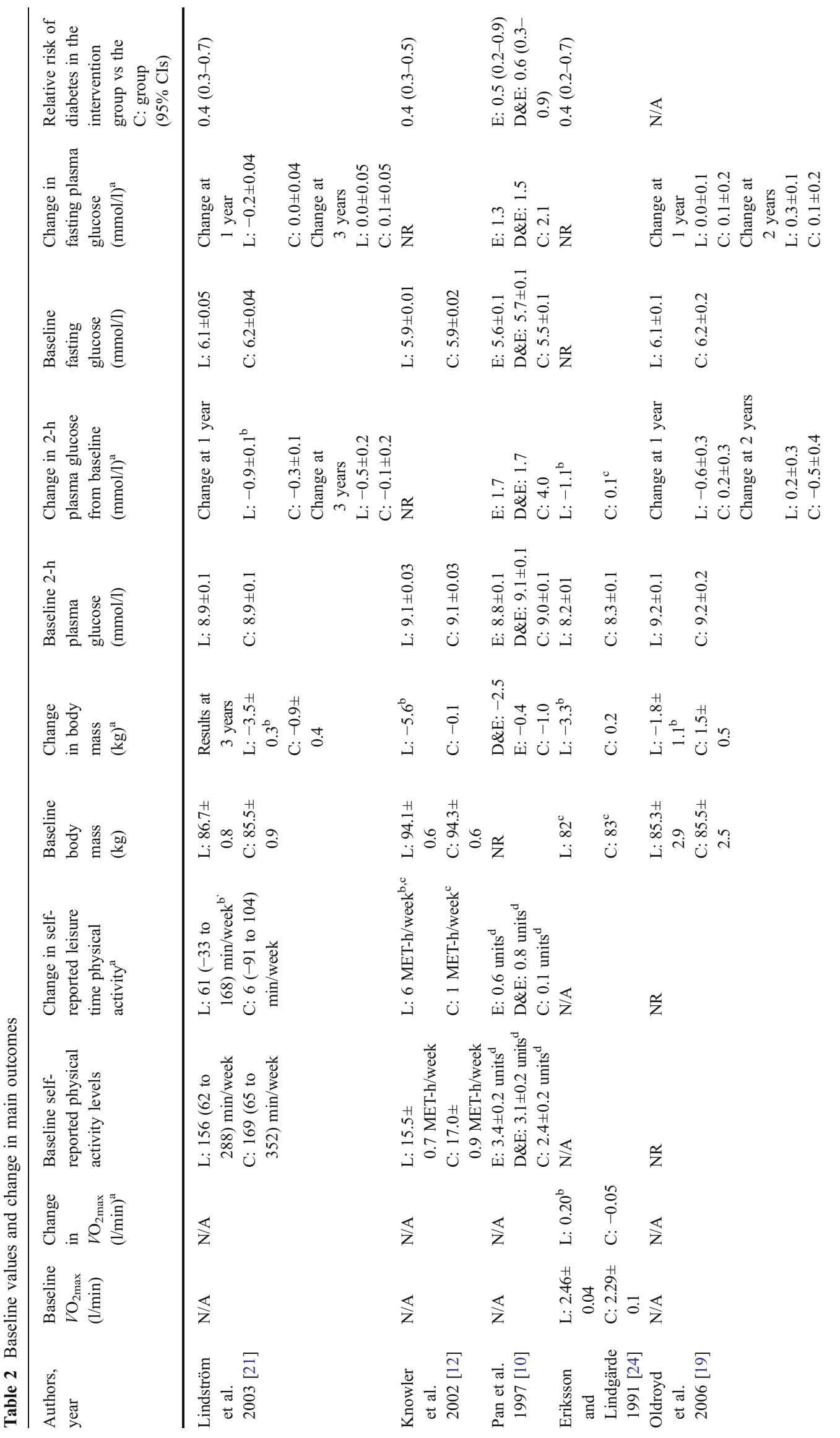




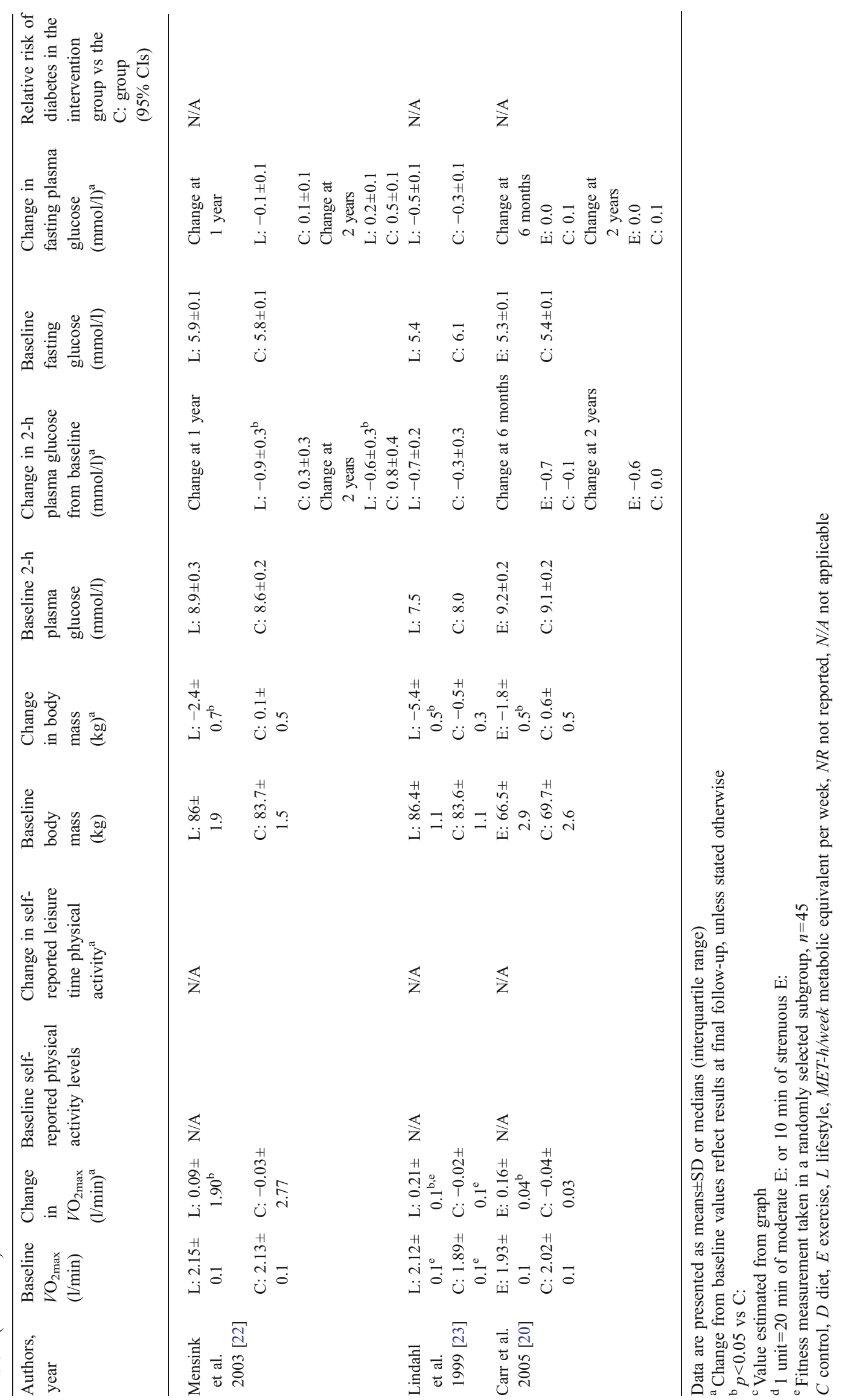


$20,22,23]$. All the studies using the incidence of diabetes as their main outcome were based on a multi-component lifestyle intervention (see intervention conditions).

Incidence of diabetes and physical activity All four of the intervention studies that measured the incidence of diabetes as their primary outcome found a significant reduction in the incidence of type 2 diabetes in the intervention group. Diabetes incidence was reduced by $42-63 \%$ in this group compared with the control group (see Table 2). The study that investigated the effect of diet and physical activity both separately and in combination found a greater reduction in the incidence of diabetes ( $46 \%$ reduced risk) in the physical activity-only group than in either the combined physical activity and diet group ( $42 \%$ reduced risk) or the diet-only group (13\% reduced risk), although the difference between groups was not statistically significant [10]. Three of these four studies relied on self-reported measures of physical activity $[10,12,21]$, and of these, only the Diabetes Prevention Program (DPP) [12] and the Finnish Diabetes Prevention Study (FDPS) [21] reported using a validated physical activity questionnaire. All three of the studies relying on self-reported physical activity levels reported non-significant to small changes in physical activity levels in the intervention group. For example, the DPP reported a mean increase in energy expenditure due to leisure time physical activity of around six metabolic equivalent hours per week [12], which is approximately equivalent to walking at a moderate pace for $15 \mathrm{~min} /$ day [25]. The FDPS reported no significant change in total physical activity levels compared with the control group and an increase of $9 \mathrm{~min} /$ day in moderate to vigorous physical activity [21], and the Da Qing IGT and Diabetes Study reported no significant change in physical activity levels compared with the control group [10]. The Malmö Feasibility Study, which used an objective outcome measure (cardiovascular fitness), reported an $8 \%$ increase in maximal oxygen uptake [24].

2-h post-challenge plasma glucose and physical activity Three of the studies that used the incidence of diabetes as their primary outcome measure also measured 2-h plasma glucose before and after the intervention [10, 21, 24]. The FDPS reported a $0.9 \mathrm{mmol} / 1$ decrease in 2-h plasma glucose after 1 year, but no significant change after 3 years [21]; the Da Qing IGT and Diabetes Study found that 2-h plasma glucose increased in all groups, but the increase in the control group was over twice that in either of the intervention groups [10]; and the Malmö Feasibility Study reported a $1.1 \mathrm{mmol} / 1$ reduction in 2 -h plasma glucose in the intervention group [24]. Of the three lifestyle intervention studies that used 2-h plasma glucose levels rather than the incidence of diabetes as the primary indictor of improved glucose tolerance [19, 22, 23], only one reported a significant difference between the groups in terms of 2-h plasma glucose at follow-up [22]. Two of the studies used a measure of cardiovascular fitness as an indicator of physical activity levels [22, 23], and one [19] used distance walked in a shuttle test [26] as a measure of physical activity. Two studies found a small to moderate increase in cardiovascular fitness $(<10 \%$ increase compared with baseline value) $[22$, $23]$, and the study using the shuttle test reported no change in the distance walked during the test [19]. Similarly, the moderate increases in cardiovascular fitness observed in the structured exercise training study were not associated with significant improvements in 2-h plasma glucose compared with the control group [20].

Fasting glucose None of the included studies reported a significant change in fasting glucose in the intervention group compared with the control group at follow-up. One study did not report fasting glucose values [24].

\section{Discussion}

Eight controlled trials in individuals with IGT were included in this review. Four studies measured the incidence of diabetes as a primary outcome measure, and found that the risk of diabetes was reduced by approximately $50 \%$ (range $42-63 \%$ ) in individuals who were encouraged to reduce their body mass through changes in diet and physical activity [10, $12,21,24]$. Although the promotion of physical activity was an important component of these studies, the effect of exercise independent of other factors on the risk of diabetes in individuals with IGT is still unclear. All but one [10] of the studies included in this review reported significant weight loss among participants. Given that weight loss is known to improve many of the factors associated with IGT, including insulin sensitivity and glycaemic control [27], and considering only modest increases in physical activity were found in these studies, the success of these interventions is likely to be largely explained by weight loss. The apparent success of the exercise-only intervention in the Da Qing IGT and Diabetes Study [10] is likely to be attributable, at least in part, to the significantly higher levels of physical activity at baseline in the exercise intervention group compared with the control group. The separation of physical activity and weight loss may seem an overcorrection given that increased physical activity may encourage weight loss through increased energy expenditure; however, several meta-analyses of controlled trials investigating the effect of physical activity on glycaemic control in individuals with diabetes found that exercise training was not associated with weight loss [28, 29]. Furthermore it is increasingly recognised that at least $60 \mathrm{~min} /$ 
day of moderate intensity exercise should be undertaken for the effective management of body mass [30], an amount that none of the interventions included in this review achieved.

Three of the four studies that investigated the effect of a lifestyle intervention in individuals with IGT on the incidence of type 2 diabetes [10-12] relied on self-reported measures of physical activity. Given the limitations of subjective measures of physical activity, particularly when measuring non-structured forms of moderate physical activity such as walking activity [31], these lifestyle intervention studies provide uncertain information about the effect of physical activity in individuals with IGT.

Results from the lifestyle intervention studies that relied on changes in 2-h plasma glucose rather than the incidence of diabetes as the primary measure of glucose control were inconclusive $[19,20,23]$. Two of the three studies were unsuccessful at improving glucose tolerance [19, 23]. Similarly, the one study that used an aerobic exercise training protocol found no improvements in glucose tolerance as measured by 2-h plasma glucose [20]. However, it did find a significant improvement in insulin sensitivity at both 6 and 24 months. This suggests that, although the intervention goal of $3 \mathrm{~h} /$ week of moderate intensity exercise was enough to improve insulin sensitivity, it was not long enough and/or of sufficient intensity to elicit the necessary magnitude of change in insulin sensitivity for this to be translated into a significant reduction in 2-h plasma glucose.

Overall, non-significant results were seen in all but two of the studies that measured 2-h plasma glucose before and after the intervention. However, despite the link between 2-h plasma glucose and diabetes risk, it does not follow that the risk of diabetes was unchanged in these studies, as demonstrated by the FDPS, which reported a non-significant change in 2-h plasma glucose over the course of the intervention but a $>50 \%$ reduction in the risk of diabetes [21]. One reason for this discrepancy is likely to be the poor repeatability of 2-h plasma glucose values [32], and given the relatively small sample sizes in most of these studies, it is possible that improvements in glucose tolerance were not detected using 2-h plasma glucose. The glucose AUC has been identified as a more reliable measure of glucose tolerance than 2-h plasma glucose [33], and is therefore a more sensitive measure of glucose tolerance. One study included in this review measured both 2-h plasma glucose and glucose AUC at baseline and follow-up [20]. It reported that, although 2-h plasma glucose did not change significantly at any of the follow-up time points, there was a significant reduction in the glucose AUC at 6 months, and a trend towards significance at 24 months.

Given the failure of the lifestyle interventions to substantially increase physical activity levels, and the inconclusive result of the structured exercise training study, the role of physical activity independent of other lifestyle changes in the treatment of prediabetes remains equivocal. However, statistical analysis of the independent effects of physical activity, which has been carried out on some of the lifestyle intervention studies included in this review, show interesting results. For example, the conclusion of the Malmö Feasibility Study that cardiovascular fitness and weight loss were equally correlated to improved glucose tolerance is supported by data from the Study on Lifestyle Intervention and Impaired Glucose Tolerance Maastricht (SLIM) [34], and a recent analysis of data from the FDPS found a $49 \%$ difference in the risk of diabetes, after adjustment for changes to body mass and diet, when comparing those in the highest and lowest tertiles of moderate to vigorous leisure time physical activity change [35]. Thus, although the overall evidence for the independent effect of physical activity in the management of prediabetes is equivocal, encouraging evidence is starting to emerge in support of the importance of exercise.

Given the limitations of the studies included in this review it is not possible to make any recommendations as to the intensity and duration of exercise needed to improve glucose tolerance and/or reduce the risk of diabetes in individuals with IGT, independently of other lifestyle changes. The equivocal nature of the evidence is reflected in the advice given by the ADA, which recommends that individuals with IGT should include $150 \mathrm{~min} /$ week of moderate to vigorous intensity exercise as part of a weight management programme [36]. However, the aforementioned analysis of the change in physical activity in the FDPS found that a difference of $246 \mathrm{~min}$ /week in median values between those in the lowest and the highest tertiles of moderate to vigorous physical activity change was associated with a significant reduction in the risk of diabetes, after adjusting for changes in diet and body mass. However, the difference of $120 \mathrm{~min} /$ week in median values between the lowest and middle tertiles was not associated with a reduced risk of diabetes [35]. Although this result was obtained by analysing the pooled cohort, and therefore provides little information about the effectiveness of the intervention itself, it does suggest that $150 \mathrm{~min} /$ week of moderate to vigorous intensity exercise is unlikely to be enough to significantly reduce the risk of type 2 diabetes in individuals with IGT, independently of other lifestyle changes. However, given that this analysis relied on selfreported physical activity levels, further rigorous studies are needed to confirm this.

All studies included in this review selected individuals using IGT as an inclusion criteria. Therefore, any conclusions from this review can only be applied to individuals with IGT and it is impossible to determine whether or not exercise may be effective in treating individuals with isolated IFG. However as individuals with isolated IFG account for a 
minority of individuals with prediabetes [37], conclusions about the effect of exercise on IGT drawn from this review will apply to the majority of individuals with prediabetes.

In summary, the majority of studies identified for this review used interventions that encouraged dietary and physical activity to initiate and maintain weight loss in individuals with IGT. Analysis of these studies found that the independent effect of physical activity in reducing the risk of type 2 diabetes in individuals with prediabetes is equivocal. Furthermore, given the limited evidence, no definite conclusion can be drawn either as to the amount of physical activity needed to reduce the risk of diabetes in individuals with prediabetes or the effectiveness of a singlecomponent physical activity intervention compared with more conventional multi-component interventions.

Thus, more evidence from rigorously designed randomised controlled trials with objective measures of physical activity is needed. As the majority of studies promoting lifestyle changes included in this review failed to substantially increase physical activity levels, strategies for effecting increased physical activity in this population also need to be researched thoroughly. Further investigation is also needed into whether exercise is equally effective in treating the different phenotypes of IGT and IFG.

Duality of interest There was no duality of interest in the writing of this review.

\section{References}

1. Amos AF, McCarty DJ, Zimmet P (1997) The rising global burden of diabetes and its complications: estimates and projections to the year 2010. Diabet Med 14(Suppl 5):S1-S85

2. Expert Committee on the Diagnosis and Classification of Diabetes Mellitus (2003) Report of the expert committee on the diagnosis and classification of diabetes mellitus. Diabetes Care 26(Suppl 1): S5-S20

3. Edelstein SL, Knowler WC, Bain RP et al (1997) Predictors of progression from impaired glucose tolerance to NIDDM: an analysis of six prospective studies. Diabetes 46:701-710

4. Eschwege E, Richard JL, Thibult N et al (1985) Coronary heart disease mortality in relation with diabetes, blood glucose and plasma insulin levels. The Paris prospective study, ten years later. Horm Metab Res Suppl 15:41-46

5. Kuller LH, Velentgas P, Barzilay J, Beauchamp NJ, O’Leary DH, Savage PJ (2000) Diabetes mellitus: subclinical cardiovascular disease and risk of incident cardiovascular disease and all-cause mortality. Arterioscler Thromb Vasc Biol 20:823-829

6. Unwin N, Shaw J, Zimmet P, Alberti KG (2002) Impaired glucose tolerance and impaired fasting glycaemia: the current status on definition and intervention. Diabet Med 19:708-723

7. Kriska AM, Blair SN, Pereira MA (1994) The potential role of physical activity in the prevention of non-insulin-dependent diabetes mellitus: the epidemiological evidence. Exerc Sport Sci Rev 22:121-143
8. Manson JE, Nathan DM, Krolewski AS, Stampfer MJ, Willett WC, Hennekens CH (1992) A prospective study of exercise and incidence of diabetes among US male physicians. JAMA 268:63-67

9. Hu FB, Manson JE, Stampfer MJ et al (2001) Diet, lifestyle, and the risk of type 2 diabetes mellitus in women. N Engl J Med 345:790-797

10. Pan XR, Li GW, Hu YH et al (1997) Effects of diet and exercise in preventing NIDDM in people with impaired glucose tolerance. The Da Qing IGT and diabetes study. Diabetes Care 20:537-544

11. Tuomilehto J, Lindström J, Eriksson JG et al (2001) Prevention of type 2 diabetes mellitus by changes in lifestyle among subjects with impaired glucose tolerance. N Engl J Med 344:1343-1350

12. Knowler WC, Barrett-Connor E, Fowler SE et al (2002) Reduction in the incidence of type 2 diabetes with lifestyle intervention or metformin. N Engl J Med 346:393-403

13. Kosaka K, Noda M, Kuzuya T (2005) Prevention of type 2 diabetes by lifestyle intervention: a Japanese trial in IGT males. Diabetes Res Clin Pract 67:152-162

14. Ramachandran A, Snehalatha C, Mary S et al (2006) The Indian diabetes prevention programme shows that lifestyle modification and metformin prevent type 2 diabetes in Asian Indian subjects with impaired glucose tolerance (IDPP-1). Diabetologia 49:289-297

15. WHO Study Group on Diabetes (1985) Diabetes mellitus: report of a WHO study group. WHO technical report series no. 727 . WHO, Geneva

16. Alberti KG, Zimmet PZ (1998) Definition, diagnosis and classification of diabetes mellitus and its complications. Part 1: diagnosis and classification of diabetes mellitus provisional report of a WHO consultation. Diabet Med 15:539-553

17. Expert Committee on the Diagnosis and Classification of Diabetes Mellitus (1997) Report of the expert committee on the diagnosis and classification of diabetes mellitus. Diabetes Care 20:1183-1197

18. Genuth S, Alberti KG, Bennett $P$ et al Expert Committee on the Diagnosis and Classification of Diabetes Mellitus (2003) Followup report on the diagnosis of diabetes mellitus. Diabetes Care 26:3160-3167

19. Oldroyd JC, Unwin NC, White M, Mathers JC, Alberti KG (2006) Randomised controlled trial evaluating lifestyle interventions in people with impaired glucose tolerance. Diabetes Res Clin Pract $72: 117-127$

20. Carr DB, Utzschneider KM, Boyko EJ et al (2005) A reduced-fat diet and aerobic exercise in Japanese Americans with impaired glucose tolerance decreases intra-abdominal fat and improves insulin sensitivity but not beta-cell function. Diabetes 54:340-347

21. Lindström J, Louheranta A, Mannelin M et al (2003) The Finnish Diabetes Prevention Study (DPS): lifestyle intervention and 3-year results on diet and physical activity. Diabetes Care 26:3230-3236

22. Mensink M, Blaak EE, Corpeleijn E, Saris WH, de Bruin TW, Feskens EJ (2003) Lifestyle intervention according to general recommendations improves glucose tolerance. Obes Res 11:1588-1596

23. Lindahl B, Nilsson TK, Jansson JH, Asplund K, Hallmans G (1999) Improved fibrinolysis by intense lifestyle intervention. A randomized trial in subjects with impaired glucose tolerance. J Intern Med 246:105-112

24. Eriksson KF, Lindgärde F (1991) Prevention of type 2 (noninsulin-dependent) diabetes mellitus by diet and physical exercise. the 6-year Malmö feasibility study. Diabetologia 34:891-898

25. Ainsworth BE, Haskell WL, Whitt MC et al (2000) Compendium of physical activities: an update of activity codes and MET intensities. Med Sci Sports 32:498S-516S

26. Singh SJ, Morgan MD, Scott S, Walters D, Hardman AE (1992) Development of a shuttle walking test of disability in patients with chronic airways obstruction. Thorax 47:1019-1024

27. American Diabetes Association (2002) Evidence-based nutrition principles and recommendations for the treatment and prevention of diabetes and related complications. Diabetes Care 25:202-212 
28. Boulé NG, Haddad E, Kenny GP, Wells GA, Sigal RJ (2001) Effects of exercise on glycemic control and body mass in type 2 diabetes mellitus: a meta-analysis of controlled clinical trials. JAMA 286:1218-1227

29. Thomas DE, Elliott E, Naughton G (2006) Exercise for type 2 diabetes mellitus. Cochrane Database Syst Rev 3:CD002968

30. Brooks GA, Butte NF, Rand WM, Flatt JP, Caballero B (2004) Chronicle of the institute of medicine physical activity recommendation: how a physical activity recommendation came to be among dietary recommendations. Am J Clin Nutr 79:921S-930S

31. Cooper AR (2003) Objective measurement of physical activity. In: MeKenna J, Riddoch C (eds) Perspectives on health and exercise. Palgrave and Macmillian, London, pp 83-108

32. Ko GT, Chan JC, Woo J et al (1998) The reproducibility and usefulness of the oral glucose tolerance test in screening for diabetes and other cardiovascular risk factors. Ann Clin Biochem 35:62-67
33. Feskens EJ, Bowles CH, Kromhout D (1991) Intra- and interindividual variability of glucose tolerance in an elderly population. J Clin Epidemiol 44:947-953

34. Mensink M, Feskens EJ, Saris WH, De Bruin TW, Blaak EE (2003) Study on Lifestyle Intervention and Impaired Glucose Tolerance Maastricht (SLIM): preliminary results after one year. Int J Obes Relat Metab Disord 27:377-384

35. Laaksonen DE, Lindström J, Lakka TA et al (2005) Physical activity in the prevention of type 2 diabetes: the Finnish diabetes prevention study. Diabetes 54:158-165

36. Sigal RJ, Kenny GP, Wasserman DH, Castaneda-Sceppa C, White RD (2006) Physical activity/exercise and type 2 diabetes: a consensus statement from the American Diabetes Association. Diabetes Care 29:1433-1438

37. Benjamin SM, Valdez R, Geiss LS, Rolka DB, Narayan KM (2003) Estimated number of adults with prediabetes in the US in 2000: opportunities for prevention. Diabetes Care 26:645-649 